\title{
INTERFERÓN TAU EN LA VENTANA DE RECONOCIMIENTO MATERNO EMBRIONARIO BOVINO
}

\section{INTERFERON TAU IN THE BOVINE MATERNAL-EMBRYONIC RECOGNITION WINDOW}

\author{
Yasser Lenis, MVZ, Esp, M.Sc. (c)ㅎ Nicolás Ramón, MV, M.Sc. (c)²; Juan Restrepo, MV, Esp, M.Sc. (c) ${ }^{3}$; \\ Martha Olivera, M.V, Dr. Cien. Agr. ; Ariel Tarazona, Zoot, M.Sc. ${ }^{5}$
}

\begin{abstract}
${ }^{1}$ Candidato a Magister en Ciencias Animales, Universidad de Antioquia, Facultad de Ciencias Agrarias, Joven Investigador COLCIENCIAS. Grupo BIOGÉNESIS. Carrera 75 \# 65-87 Bloque 46 Of. 321 Medellín - Colombia. e-mail: yaserudea@ gmail.com ${ }^{2}$ Candidato a Magister en Ciencias Animales, Universidad de Antioquia, Facultad de Ciencias Agrarias, Grupo BIOGÉNESIS. e-mail: nickramon@hotmail.com ${ }^{3}$ Universidad de Antioquia, Facultad de Ciencias Agrarias, Grupo BIOGÉNESIS. e-mail: juanrb66@gmail.com4 Universidad de Antioquia, Facultad de Ciencias Agrarias, Grupo BIOGÉNESIS. e-mail: syngamia@gmail.com ${ }^{5}$ Universidad Nacional de Colombia, Sede Medellín Facultad de Ciencias Agropecuarias, Grupo BIOGÉNESIS, Calle 59a \# 63-20 Bloque 50 Of. 316 Medellín - Colombia. e-mail: arielmarcel@gmail.com.
\end{abstract}

Rev. U.D.C.A Act. \& Div. Cient. 13 (1): 17-28, 2010

\section{RESUMEN}

El reconocimiento materno embrionario bovino es uno de los eventos de mayor importancia en la reproducción. Este proceso está regulado por múltiples señales celulares y endocrinas, entre el embrión, el endometrio y el cuerpo lúteo, que constituye la glándula transitoria de gran trascendencia en la ventana de reconocimiento materno embrionario, por ser el responsable de la producción de la progesterona. El interferón trofoblástico bovino (bINT-T) llamado así por el sitio de producción, es la principal señal para el éxito en el establecimiento de la preñez, que favorece los procesos luteotrópicos funcionales y estructurales, garantizando la producción de progesterona y la integridad de las células, que constituyen el cuerpo lúteo. La luteólisis es un proceso comprendido en dos etapas: la luteólisis funcional y la estructural, ambas mediadas, principalmente, por la $\mathrm{PGF}_{2} \alpha$. Este proceso es necesario para que el estro se presente de forma cíclica; una vez iniciados los mecanismos luteolíticos la posibilidad de una gestación se ve reducida. Dentro de los principales efectos del bINT-T esta la inhibición de la síntesis y la liberación, de forma pulsátil, de la $\mathrm{PGF}_{2} \alpha$ en el endometrio bovino, el cual, está constituido por dos tipos de células: las endometriales epiteliales (CEEP) y las endometriales estromales (CEES); las primeras, poseen la capacidad de producir entre el 60 al $70 \%$ de la $\mathrm{PGF}_{2} \alpha$ total. El propósito de esta revisión es describir el papel del bINT-T en la ventana de reconocimiento materno embrionario y sus principales efectos sobre la síntesis y la secreción de $\mathrm{PGF}_{2} \alpha$ y $\mathrm{PGE}_{2}$, en el endometrio bovino

Palabras clave: Embrión, luteólisis, luteotropismo, trofoblasto.

\section{SUMMARY}

Maternal-embryonic recognition is one of the most important events in bovine reproduction. This process is regulated by multiple cellular and endocrine signals among the embryo, the endometrium and corpus luteum, the most important transitory gland in the maternal-embryonic recognition window since it is responsible for the production of progesterone. Bovine trophoblastic interferon (bINT-T) named after the site of production (embryonic trofoectoderm) is the main signal for the successful establishment of pregnancy. bINT-T, 
supports functional and structural luteotropic process ensuring the production of progesterone and integrity of the cells forming the corpus luteum. Luteolysis is divided in two stages: Functional and structural luteolysis, both mediated primarily by $\mathrm{PGF}_{2} \alpha$. Luteolysis is necessary for maintenance of the estrous cycle cyclicity. Once luteolitic mechanisms initiate, the chances of maintaining the pregnancy are reduced. Among the main efects of bINT-T, are the inhibition of the synthesis and pulsatile release of $\mathrm{PGF}_{2} \alpha$ in the bovine endometrium which consists of two types of cells: endometrial epithelial cells (CEEP) and endometrial stromal cells (CEES). The former produces between 60 to $70 \%$ of the total $\mathrm{PGF}_{2} \alpha$. The purpose of this review is to describe the role of bINT-T in the maternal-embryonic recognition window and its major effects on the synthesis and secretion of $\mathrm{PGE}_{2}$ and $\mathrm{PGF}_{2} \alpha$ in the bovine endometrium.

Key words: Embryo, luteolysis, luteotropism, trofoblast.

\section{INTRODUCCIÓN}

El interferón-T (INT-T), también conocido como interferón trofoblástico, fue descubierto mediante el cultivo, aislamiento y purificación de extractos de blasctocistos ovinos, que secretaban una proteína con propiedades antiluteolíticas y que prolongaban la fase lútea de la hembra (Roberts, 2007; Asselin et al. 1997). En 1987, se identificó la secuencia aminoacídica, lo que permitió sintetizarlo, de manera exitosa, para la investigación en ciencias básicas (Demmers et al. 2001). En la actualidad, es producido de forma recombinante y se han reconocido diferentes isoformas del mismo. Dentro de las diversas especies rumiantes existen otras isoformas de interferones trofoblásticos, y entre las más estudiadas están: el interferón trofoblástico ovino (OINT-T) y el interferón trofoblástico bovino (bINT-T) y, en menor medida, los interferones trofoblásticos de especies como la jirafa y otros rumiantes (Weems et al. 2006; Walker et al. 2009).

Uno de los hallazgos más relevantes fue la identificación de distintas isoformas de INT-T dentro de una misma especie; en el bovino, al menos, once a doce distintos interferones trofoblásticos han sido identificados de embriones cultivados in-vitro (Hashizume et al. 2006). Lo anterior podría sugerir la capacidad de un embrión de inhibir, mediante diferentes rutas moleculares, los procesos luteolíticos y así garantizar los procesos de reconocimiento e implantación embrionaria (Walker et al. 2009).

El bINT-T, por su parte, participa en el establecimiento y reconocimiento de la gestación, proceso regulado por múltiples señales moleculares e interacciones celulares, principalmente, entre el embrión y el cuerpo lúteo (CL) (Arosh et al. 2004; Asselin et al. 1997). Durante los días 15 al 17 del ciclo estral, la viabilidad embrionaria juega un papel importante para inhibir los procesos luteolíticos, favoreciendo la implantación y el mantenimiento de la gestación. Este periodo es considerado crítico para garantizar los procesos luteoprotectivos, mediante la modulación de la producción de la prostaglandina $\mathrm{F}_{2}$ alpha $\left(\mathrm{PGF}_{2} \alpha\right)$ y prostaglandina $\mathrm{E}_{2}\left(\mathrm{PGE}_{2}\right)$, en el endometrio bovino (Tithof et al. 2007; Weems et al. 2006; Fortier et al. 1988).

La pérdida funcional y estructural del CL es llevada acabo por la $\mathrm{PGF}_{2} \alpha$ que es el principal factor luteolítico y es producida, primordialmente, en el endometrio bovino (Acosta et al. 2008; Asselin et al. 1996; Fortier et al. 1988), el cual, a su vez, se encuentra constituido por dos biotipos celulares: las células endometriales epiteliales bovinas (CEEP) y las células endometriales estromales bovinas (CEES). Las primeras son las mayores responsables de los procesos luteolíticos, ya que sintetizan $\mathrm{PGF}_{2} \alpha$ en mayor cantidad que $\mathrm{PGE}_{2}$, mientras que las CEES son las responsables de favorecer los procesos luteotrópicos, debido a que producen más $\mathrm{PGE}_{2}$ que $\mathrm{PGF}_{2} \alpha$ (Skarzynski et al. 2008; Fortier et al. 1988) (Gráfica 1).

Durante la ventana de reconocimiento materno embrionario, los niveles de $\mathrm{PGE}_{2}$ aumentan respecto los de $\mathrm{PGF}_{2} \alpha$, favoreciendo los efectos luteoprotectivos como: vasodilatación, angiogénesis, quiescencia uterina, receptividad uterina, entre otros. Este aumento, se debe principalmente a la síntesis y secreción por parte de las células trofoectodérmicas del embrión del bINT-T, que se secreta en altas cantidades, entre los días 13 al 17; sin embargo, los niveles permanecen altos en promedio hasta el día 27 de gestación (Arosh et al. 2004; Tithof et al. 2007; Skarzynski et al. 2008).

El mecanismo de acción del bINT-T involucra la unión a su receptor, que se encuentra ubicado en la membrana celular de las CEEP y las CEES, favoreciendo la síntesis 
y la secreción de $\mathrm{PGE}_{2}$, mediante mecanismos celulares específicos, como el aumento del mARN, que codifica para la enzima prostaglandina $E$ sintasa, responsable de la síntesis de la $\mathrm{PGE}_{2}$ (Hashizume et al. 2006).

Una de las principales causas para la pérdida embrionaria temprana es la respuesta inadecuada en el endometrio ante el INT-T, debido a un aumento de la síntesis y la secreción en pulsos de la $\mathrm{PGF}_{2} \alpha$, que induce los procesos luteolíticos (Roberts et al. 2008; Spencer et al. 2004; Arosh et al. 2004; Parent et al. 2003a; Tithof et al. 2007; Olivera et al. 2007).

Aunque el mecanismo de acción del bINT-T se ha estudiado a fondo, todavía quedan algunos vacíos de cómo podría modular la producción de $\mathrm{PGF}_{2} \alpha$ y $\mathrm{PGE}_{2}$ en las CEEP y las CEES. El propósito de esta revisión es describir el papel del INT-T en la ventana de reconocimiento materno embrionario y su función en la regulación de la síntesis de $\mathrm{PGF}_{2} \alpha$ y $\mathrm{PGE}_{2}$.

\section{GENERALIDADES DE LOS INTERFERONES}

En 1965, se reportó por primera vez los efectos de los interferones, cuando estas proteínas interfirieron en un cultivo de leucocitos humanos, en el crecimiento viral, evitando la replicación de partículas virales en el medio celular y en las células sometidas a cultivo (Billiau $\mathcal{E}$ Matthys, 2009).

Los interferones son proteínas producidas por el sistema inmunitario de la mayoría de los animales, como respuesta a agentes infecciosos o extraños, como virus, bacterias, parásitos, células cancerígenas, entre otros. La gran mayoría de los interferones pertenecen a la clase de las glicoproteínas, ya que su estructura cuenta con azúcares específicos, que confieren características bioquímicas, como su grado de solubilidad en medios acuosos (Billiau E Matthys, 2009; Gómez et al. 2007; Demartini et al. 2003). Existen, al menos, dos biotipos de interferones (biotipo I y II), que fueron clasificados según su estructura y su origen celular con letras griegas. Los principales interferones tipo I son: $\alpha, \beta, \omega, \mathrm{T}$, y, en el caso del tipo II, está el interferón $\gamma$ (INT- $\gamma$ ), el cual, es codificado por un gen inducible y, a pesar de considerarse un producto primario de linfocitos $\mathrm{T}$ para activar macrófagos, es liberado por una amplia variedad de tipos celulares, cumpliendo diferentes actividades biológicas (Spencer et al. 2004. Demartini et al. 2003, Parent et al. 2003a).
Investigaciones en la década de los 80 lograron, como objetivo la purificación de una proteína producida y secretada por células trofoblásticas de un embrión ovino, que tenía como principal función fisiológica favorecer la presentación de los efectos antiluteolíticos. Posteriores investigaciones permitieron corroborar que dicha proteína se trataba de un biotipo de interferón que denominaron interferón trofoblástico (Demmers et al. 2001).

Al principio el interferón trofoblástico fue clasificado dentro del subgrupo 0 , en razón de su similitud con el interferón $\alpha$, que alcanza un $70 \%$ de igualdad en su identidad aminoacídica; sin embargo, la analogía entre bIFNT y olFNT es mayor, por ellos varias investigaciones utilizaron el olFNT, para responder preguntas planteadas en células endometriales bovinas, como un modelo in vitro. Estas observaciones han llevado a clasificar a los interferones trofoblásticos como un nuevo subtipo dentro de los interferones de tipo I, denominados tau (Weems et al. 2006).

La isoforma del bIFNT, expresado solo en el trofoblasto de embriones rumiantes, representa una de las cinco familias relacionadas con los interferones tipo I, denominados INT $\alpha, \beta, \omega$ y T. Dentro de los biotipos T encontramos, principalmente, el bIFNT y olFNT, cuyas diferencias estructurales son pocas (Roberts et al. 2008; Demmers et al. 2001).

Análisis filogenéticos han evidenciado que los interferones tipo I y II son conservados evolutivamente y divergieron de un gen precursor, hace más de 250 millones de años, en todas las especies estudiadas hasta el momento; no obstante, los biotipos de los interferones trofoblásticos fueron originados más recientemente en los rumiantes, a partir de la expresión de genes propios activados, hace más de 30 millones de años (Roberts, 2007; Demmers et al. 2001; Christine et al. 2009).

Gran parte de los genes que inducen síntesis de factores o moléculas relacionadas con la reproducción en la vaca son expresados en la placenta. Estos genes codifican su producto, mediante la activación de señales intracelulares específicas, sintetizando glicoproteínas asociadas a la gestación, incluyendo el IFNT, existiendo en el bovino, por lo menos tres genes distintos, que pudieran codificarlo (Christine et al. 2009). 
Existe una gran similitud en la estructura química del IFNT en rumiantes, que se basa en una alta homología de los nucleótidos que componen el ADN codificante, para esta proteína (Walker et al. 2009).

\section{PROPIEDADES BIOQUÍMICAS DEL INTEFERÓN TAU}

El IFNT posee una masa molecular entre 22.000 y 24.000kDa. Su estructura de 172 aminoácidos, se encuentra glicosilada en su extremo amino terminal con algunos oligosacáridos, confiriéndole mayor versatilidad química a su estructura, la cual, difiere del olFNT, debido a que este último no posee ninguna glicosilación en su estructura (Roberts, 2007; Demmers et al. 2001). La estructura primaría, le permite a la estructura tridimensional del IFNT formar hélices- $\alpha$, que contienen enlaces disulfuro entre aminoácidos, como la cisteína; láminas- $\beta$ plegadas y bucles irregulares, le confieren al IFNT una estructura tridimensional (Demmers et al. 2001).

El bIFNT ha sido identificado como una señal crítica en el reconocimiento materno embrionario bovino. Hasta el momento, se han identificado entre once a doce variantes polimórficas, las cuales, fueron caracterizadas mediante técnicas de laboratorio con cADN; sin embargo, no todos han transcrito, pues no ha sido posible la identificación de algunos transcriptos, lo que sugeriría la presencia de algunos genes complementarios en la células trofoectodérmicas (Roberts, 2007; Demmers et al. 2001; Binelli et al. 2001; Parent et al. 2003b; Assal et al. 1995).

Como consecuencia de las variaciones alélicas en cada una de las especies, incluyendo el bovino, el bIFNT puede presentar pequeñas variaciones en su estructura química final. Se ha logrado demostrar que estas variaciones le confieren a cada bIFNT una potencia biológica distinta, aumentando o disminuyendo algunos de los efectos luteotrópicos (Ealy et al. 1998).

El bIFNT posee la capacidad de unirse a su receptor tipo rINT1, el cual, a su vez, se subdivide en dos biotipos (rINTA1 y rINTA2) y mediante procesos bioquímicos activa la ruta de la STAT, induciendo la transcripción de genes involucrados en procesos antivirales, antiproliferativos y con efectos luteales (Roberts, 2007; Demmers et al. 2001).

\section{SÍNTESIS DEL INTERFERÓN TAU}

La síntesis y la secreción del bIFNT, se efectúa en el trofoectodermo extraembrionario del blastocisto, en su etapa preimplantatoria y alcanza niveles máximos durante los días 15 al 17, sintetizándose hasta el día 28 de gestación (Arosh et al. 2004). Entre los días 13 al 15 los niveles de mARN, para el bIFNT, son elevados en las células trofoectodérmicas, coincidiendo con la etapa de reconocimiento materno embrionario; posterior a este evento fisiológico, los niveles del bIFNT van disminuyendo de forma progresiva (Arosh et al. 2004; Roberts et al. 2008; Demmers et al. 2001).

Hasta el momento, se han descubierto un sin número de factores moleculares que podrían inducir la síntesis de bIFNT en el trofoectodermo embrionario, comprendiendo la interleuquina tipo 3 (IL3) y el factor estimulante de granulocitos y macrófagos (GM-CSF); aún así, se desconoce el papel de otros factores y las vías celulares por las cuales se induciría la síntesis del bIFNT. Se evidenció la importancia de estos factores de crecimiento y de las citoquinas inflamatorias secretadas por el endometrio, cuando se demostró que embriones producidos in vitro y transferidos a vacas receptoras incrementaban la producción de bIFNT unas 500 veces más que los embriones que eran cultivados hasta el día once, en ambiente controlado (Yamaguchi et al. 2001) (Gráfica 1).

En 1989, se demostró que la concentración del bIFNT se puede modular por el grado de desarrollo embrionario, produciendo una mayor concentración los embriones en estadío más avanzados morfológica y funcionalmente (blastocistos), que los que se encontraban en estadío de mórula. Una vez el embrión ha eclosionado y la zona trofoblástica tiene contacto establecido con el epitelio uterino, ocurre un cese en la producción de bIFNT (Demmers et al. 2001).

A diferencia del IFN $\alpha$ y IFN $\beta$, la región promotora y su "enhancer", ubicados en la región codificante para el bIFNT, no poseen la secuencia viral inductora, por lo que llevó muchos años de investigación adicional poder comprender cuáles eran las señales celulares que inducían la activación génica, que daba lugar a la síntesis del bIFNT (Ashoworth E Bazer, 1989; Roberts, 2007; Demmers et al. 2001). En 1998, mediante la transfección de una línea celular humana, se logró determinar que 


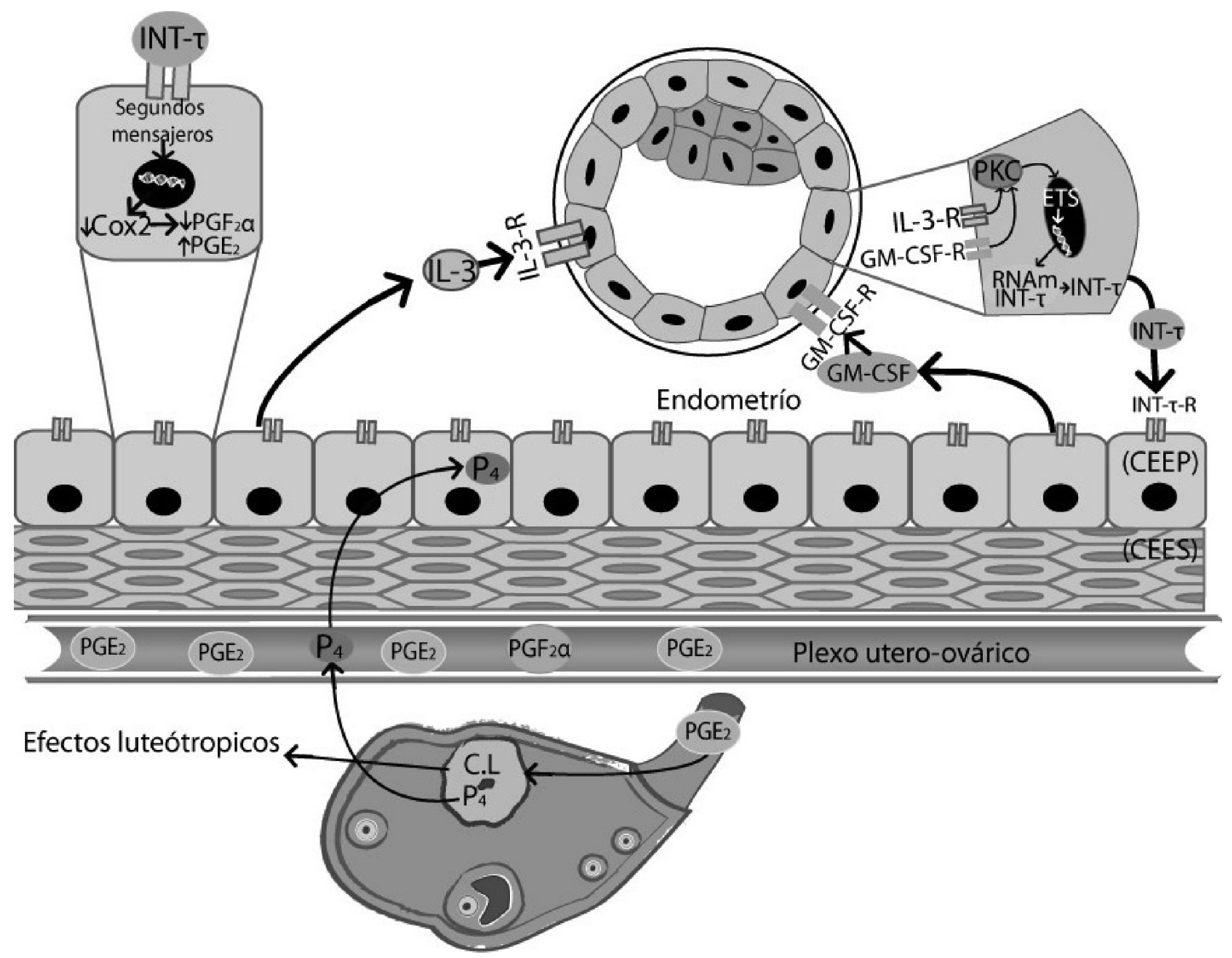

Gráfica 1: Mecanismo de acción del bIFNT en el endometrio bovino (adaptada y modificada de Yamaguchi et al. 2001).

IFNT: Interferón tau.

IFNT-R: Receptor para el interferón tau.

PKC: Proteína kinasa $C$.

$\mathrm{PGF}_{2}$ a: Prostaglandina $\mathrm{F}_{2}$ alfa.

$\mathrm{PGE}_{2}$ : Prostaglandina $\mathrm{E}_{2}$ alfa.

RNAm: Ácido Ribonucléico Mensajero.

GM-CSF: Factor Estimulante de Granulocitos y Macrófagos.

GM-CSF-R: Receptor para Factor Estimulante de Granulocitos y Macrófagos.

IL3: Interleuquina tipo 3.

IL3-R: Receptor para Interleuquina tipo 3.

CL: Cuerpo Lúteo.

P4: Progesterona.

CEEP: Células endometriales epiteliales.

CEES: Células estromales epiteliales. 
las Ets (familia de factores transcripcionales) eran las responsables de inducir la activación de los genes codificantes para el bIFNT. Las Ets regulan y activan una gran variedad de genes en diversos biotipos celulares, especialmente, en las células epiteliales, incluyendo las células trofoectodérmicas (Ezashi et al. 1998; Imakawa et al. 1993) (Gráfica 1).

Se demostró, en 1993, que la suplementación con factores de granulocito y de macrófagos del medio de cultivo para embriones aumentó la producción de proteínas que activaban los promotores de las secuencias codificantes para el bIFNT (Ezashi et al. 1998; Imakawa et al. 1995). La adición de GM-CSF al medio de cultivo de embriones de oveja, duplicó la actividad antiviral del sobrenadante respecto a los controles y aumentó la producción de oIFNT (Jo-ann et al. 2001). Estos resultados sugieren que citoquinas de origen materno pueden estar influyendo en las señales que participan en el reconocimiento materno de la preñez. Así mismo, al agregar factor de crecimiento insulínico I y II (IGF I y II) al medio de cultivo de embriones, se incrementó la síntesis de oIFNT, indicando que ciertos componentes presentes en el medio ambiente uterino, durante la preñez temprana, se requieren para la síntesis del bIFNT (Jo-ann et al. 2001).

Una vez el mARN para el bIFNT es transcrito, la traducción ocurre en los ribosomas libres de las células o en los que se encuentran acoplados al retículo endoplásmico rugoso, donde se inician los procesos de biotransformación, como la glicosilación y que es secretado, posiblemente, por exocitosis (Arosh et al. 2004; Tithof et al. 2007).

\section{MECANISMO DE ACCIÓN DEL INTERFERÓN TAU}

El receptor del bIFNT, se encuentra ubicado en la membrana plasmática de las CEEP y CEES, acoplado a proteínas de la familia Jack Kinasas, las cuales, pueden fosforilar otras proteínas, mediante residuos de tirosina (TYK 2) en sus estructuras (Roberts et al. 2008).

La ausencia de un embrión durante los días 15 al 18 del ciclo estral bovino induce un aumento de la oxitocina luteal, favoreciendo la síntesis de $\mathrm{PGF}_{2} \alpha$ y $\mathrm{PGE}_{2}$ en la CEEP y, en menor proporción, en las células luteales grandes (CLG) y células luteales pequeñas (CLP), activando la luteólisis funcional y estructural, que conlleva al retorno a un nuevo ciclo. Por el contrario, la presencia de un embrión competente, promueve el inicio de la síntesis de IFNT, que aumenta progresivamente entre los días 13 y 18 del reconocimiento materno embrionario, favoreciendo la presentación de los efectos luteotrópicos y el establecimiento de la gestación (Arosh et al. 2004; Woclawek et al. 2004; Asselin et al. 1996).

Investigaciones recientes sugieren que el INT-T activa la ruta de las Jak Kinasas (Jak-Star), para posteriormente fosforilar la STAT, ubicada en el citoplasma de las CEEP y las CEES; una vez activada la STAT es translocada al núcleo, donde activa el complejo nuclear de unión para STAT (Binelli et al. 2001).

El mecanismo de acción propuesto para el bIFNT consiste en la fosforilación de las proteínas activadoras de transcripción o STAT. La naturaleza química de los interferones no les permite ingresar a la célula blanco para ejercer un efecto fisiológico, lo que hace necesarias, la activación de proteínas intracelulares que traducen la señal y desencadenan el efecto final, mediante la inclusión de grupos fosfatos a la STAT (Demmers et al. 2001; Binelli et al. 2001; Billiau et al. 2009). Existen diferentes biotipos para la STAT (STAT1, STAT2, STAT3) ubicadas, generalmente, en el citoplasma de la célula blanco. Cada una de estas posee rutas moleculares diferentes y son activadas por estímulos específicos, como el bIFNT (Cheng et al. 2005)

Binelli et al. (2001) demostraron que diferentes concentraciones de bIFNT $(0,2 \mathrm{ng} / \mathrm{mL}, 11,6 \mathrm{ng} / \mathrm{mL}$, $12 \mathrm{ng} / \mathrm{mL}$ y $50 \mathrm{ng} / \mathrm{mL}$ ) en el medio de cultivo de CEEP, afectaba de forma individual la tasa de fosforilación de cada una de las STAT (Billiau et al. 2009), sugiriendo que la calidad del trofoblasto y su capacidad de producir bIFNT podría reducir los efectos luteotrópicos y afectar el reconocimiento materno-embrionario (Cheng et al. 2004).

Una vez el bIFNT interactúa con su receptor de membrana, se inicia la fosforilación de unidades de STAT1 de forma dosis dependiente. La STAT presenta la señal peptídica de importación nuclear y, una vez activada, se une a una proteína de unión del ADN, llamada ISGF3 $\gamma$. Este complejo, se trasloca al núcleo, donde interactúa con secuencias específicas del ADN, denominadas "elementos respondedores estimulados 
por IFNT" (Demmers et al. 2001; Binelli et al. 2001; Ashoworth E Bazer, 1989), para expresar los genes que favorecen los efectos luteotrópicos. Los segundos mensajeros generados por el estímulo del bIFNT con su receptor tienen efectos directos en la expresión génica de las CEEP y CEES. La activación de proteínas de unión específicas al ADN son las responsables de modular negativamente la traducción de enzimas relacionadas con la síntesis de $\mathrm{PGF}_{2} \alpha$ y $\mathrm{PGE}_{2}$, como la cicloxigenasa tipo 2 (Cox 2) (Kim et al. 2003), la cual, se expresa a partir de unos genes de tipo inducibles. Otra enzima regulada de forma negativa por el bIFNT es la prostaglandina $\mathrm{H}$ sintasa, relacionada directamente con el aumento en la concentración de $\mathrm{PGF}_{2} \alpha$ y $\mathrm{PGE}_{2}$, en el endometrio bovino. Estos dos tipos de genes responden a señales celulares, como las STAT (Demmers et al. 2001; Guzeloglu et al. 2004a; Arosh et al. 2002) (Gráfica 1).

\section{EFECTOS DEL INTERFERÓN TAU EN LA VENTANA DE RECONOCIMIENTO MATERNO EMBRIONARIO}

Las CEEP y las CEES poseen en su membrana plasmática el receptor IFNAR para bIFNT. Una vez activado, se desencadenan señales intracelulares, que generan diversidad de efectos fisiológicos en el útero bovino. Hasta el momento, no se ha observado ningún efecto directo de bINFT sobre el CL (Guzeloglu et al. 2004a).

Los efectos del bIFNT en el aparato reproductivo son diversos. El primero que se descubrió fue la prolongación de la fase lútea en ovejas, que eran sometidas a infusiones intrauterinas de soluciones que contenían oIFNT. Estas ovejas presentaban estructura luteal hasta 45 días después de la infusión; posteriormente, se logró demostrar que la concentración del oIFNT aplicado en el lumen uterino podía aumentar la fase lútea o, por el contrario, no afectarla (Kim et al. 2003; Chen et al. 2006; Thatcher et al. 1994; Ashoworth et al. 1989).

Durante la ventana de reconocimiento maternoembrionario (día 13 a 18 del ciclo estral), el CL puede sufrir luteólisis o seguir produciendo progesterona. El primer evento conduce al retorno a la ciclicidad, mientras que el segundo favorece los efectos luterotrópicos, necesarios para la gestación. La regulación de la luteólisis esta modulada por la interacción de varias moléculas, entre las cuales están los estrógenos, la oxitocina, la $\mathrm{PGF}_{2} \alpha$ y sus respectivos receptores (Arosh et al. 2004; Robinson et al. 2002; Rodríguez et al. 2006). Los estrógenos poseen dos biotipos de receptores tipo $\alpha$ y $\beta$, expresados de manera características en algunos órganos o aparatos, como el reproductivo (Kim et al. 2003; Farina et al. 2007). Los estrógenos sintetizados por los folículos en el ovario en crecimiento modulan la expresión del mARN para el receptor de oxitocina (OTr), principalmente, en las CEEP. Existe una relación directa entre la capacidad de los estrógenos de unirse a sus receptores y la cantidad de mARN para el OTr (Ashoworth et al. 1989). Por lo tanto, a mayor concentración de complejo estrógenos-receptor, mayor actividad génica para la expresión del OTr (Chen et al. 2006; Guzeloglu et al. 2004b).

La transcripción de los interferones tipo I es inducida mediante la activación de factores reguladores de los interferones tipo 1 (FRI 1) y tipo 2 (FRI 2). El FRI 1 induce la expresión directamente del FRI 2, pero este último es un factor supresor para la activación del FRI-1; adicionalmente, el FRI-2 tiene la capacidad de inhibir la expresión de genes específicos de respuesta viral, como el gen antígeno nuclear tipo 1 y el gen codificante para la óxido nítrico sintasa, importante en la síntesis de $\mathrm{PGF}_{2} \alpha$ y $\mathrm{PGE}_{2}$, en las CEES (Binelli et al. 2001; Woclawek et al. 2004).

El FRI-2 actúa, principalmente, como inhibidor del receptor de estrógenos (ER2 $\alpha$ ) en la CEEP, evitando la unión hormona-receptor, necesaria para la síntesis de OTr. Además, cumple su función en el endometrio evitando que se origina la producción de OTr, necesarios para la vía de producción de $\mathrm{PGF}_{2} \alpha$ (Spencer et al. 2004; Guzeloglu et al. 2004a; Demmers et al. 2001; Jean et al. 2008).

La unión de progesterona a sus receptores en el trofoblasto activa los genes de respuesta a retrovirus (JSRV), que tienen un efecto directo para que el embrión inicie la producción de INFT (Spencer et al. 2004; Arosh et al. 2004; Skarzynski et al. 2000a y b). Algunos de los ligandos para JSRV, se encuentran en la membrana de las CEEP y CEES, como también en las glándulas endometriales uterinas, lo que sugiere que la unión del embrión a la pared del endometrio es una señal para desencadenar funciones fisiológicas, como las luteotrópicas y la diferenciación y función de la placenta (Spencer et al. 2004; Arosh et al. 2004). 
Durante los días 10-12 del ciclo estral, la progesterona se une a sus receptores nucleares en las CEEP, inhibiendo la expresión de los OTr en la membrana plasmática. Este periodo se denomina "periodo de bloqueo" (Jo-ann et al. 2001). Posteriormente, la progesterona es la responsable del "down regulation" de los receptores de estrógenos, con la consiguiente disminución del ER $\alpha$, lo que lleva a una rápida disminución en la expresión de OTr (Weems et al. 2006; Chard, 1989; Olivera et al. 2007; Okuda et al. 2004).

El aumento de la expresión de genes para JSRV en el epitelio uterino, se halla altamente relacionado con los niveles de progesterona en sangre periférica y la expresión de receptores de progesterona (PR), en el epitelio endotelial. La expresión génica para los PR es baja entre los días 8-10 del diestro, aumentando entre los días 13-15, a la espera de señales celulares, generadas por un embrión viable. De no generarse el reconocimiento, la expresión y la síntesis para el PR, se disminuye al nivel mínimo basal (Spencer et al. 2004; Arosh et al. 2004; George E Lamming, 2001; Spencer et al. 1995, 1996).

El éxito o no del reconocimiento materno embrionario depende de la adecuada comodulación entre la progesterona y el bIFNT, o los estrógenos y la oxitocina, además de la expresión de los receptores específicos. Alteraciones en la señalización celular o molecular podría desencadenar la reabsorción embrionaria. Como aplicación de este conocimiento, se ha planteado la posibilidad de suministrar bIFNT, en este periodo, con la hipótesis de favorecer el proceso de reconocimiento materno embrionario. El bIFNT, se puede producir de forma recombinante (Assal et al. 1995); sin embargo, uno de los mayores inconvenientes es su consecución comercial, ya que son pocos los grupos en el mundo que lo producen y el costo es muy alto (Roberts, 2007).

Los efectos del bIFNT, se han estudiado usando como modelo hembras preñadas ovinas y bovinas, demostrando que el útero posee un bajo número de receptores funcionales para oxitocina en sus CEEP y en el miometrio. En forma similar, la infusión intrauterina de oIFNT entre los días 11-15 del ciclo estral, reduce el número de receptores para estrógenos y los OTr, y la afinidad de este último, para con su ligando (Kim et al. 2003; Spencer et al. 2004; Okuda et al. 2004; Skarzynski et al. 2000a y b).
El oIFNT puede inhibir la síntesis y la afinidad de receptores para oxitocina directa o indirectamente por un mecanismo de "down-regulation" de receptores para estrógenos y/o estabilizando los receptores para progesterona (Mark et al. 2008). De este modo, el INFT inhibiría los pulsos luteolíticos de $\mathrm{PGF}_{2} \alpha$ inducidos por oxitocina (Tithof et al. 2007; Arosh et al. 2004; Spencer et al. 1995, 1996).

Se concluye, que el bIFNT es una de las señales más importantes en la ventana de reconocimiento materno embrionario, proceso que esta regulado de manera multifactorial. Los mecanismos que potencialmente podrían ser desencadenados por el IFNT, incluyen: 1) Estabilización o "up-regulation" de receptores endometriales para progesterona, con el objeto de extender el período de bloqueo por progesterona y, de este modo, evitar la expresión de receptores para estrógenos y/o receptores para oxitocina. 2) Inhibición directa de receptores para estrógenos. 3) Inhibición directa de la síntesis de receptores para oxitocina evitando la liberación pulsátil de PGF2 $\alpha$ (Tithof et al. 2007; Arosh et al. 2004; Jo-ann et al. 2001). No obstante, se evidencia que aún existen muchos vacíos en el conocimiento, principalmente, en la actualización de nuevas técnicas de laboratorio, que permitan la síntesis de manera rápida y económica del bIFNT, para facilitar las investigaciones básicas, para aproximarnos al entendimiento de los principales procesos moleculares que están ocurriendo en el aparato reproductivo y desarrollar nuevas terapias específicas, con las cuales, se pudiera mejorar el ambiente uterino y, así, favorecer los indicadores reproductivos, como: el porcentaje de preñez, intervalos entre partos, servicios por concepción, entre otros.

\section{BIBLIOGRAFÍA}

1. ACOSTA, T.J.; BAH, M.M.; KORZEKWA, A.; WOCLAWEK-POTOCKA, I., MARKIEWICZ, W.; JAROCZEWSKI, J.J.; OKUDA, K.; SKARZYNSKI, D.J. 2008. Acute Changes in Circulating Concentrations of Progesterone and Nitric Oxide and Partial Pressure of Oxygen During Prostaglandin F2alpha-induced Luteolysis in Cattle. J. Reprod. Dev. 55(2):149-155.

2. AROSH, J.A.; BANU, S.K.; CHAPDELAINE, P.; MADORE, E.; SIROIS, J.; FORTIER, M.A. 2004 May 
Prostaglandin biosynthesis, transport, and signaling in corpus luteum: a basis for autoregulation of luteal function. Endocrinology. 145(5):2551-2560.

3. AROSH, J.A.; PARENT, J.; CHAPDELAINE, P.; SIROIS, J.; FORTIER, M.A. 2002. Expression of cyclooxygenases 1 and 2 and prostaglandin $E$ synthase in bovine endometrial tissue during the estrous cycle. Biol. Reprod. 1:161-169.

4. AROSH, J.A.; BANU, S.K.; KIMMINS, S.; CHAPDELAINE, P.; MACLAREN, L.A.; FORTIER, M.A. 2004. Effect of interferon $T$ on prostanglandin biosintesis, transport, and signaling at the time of maternal recognition of pregnancy in cattle: evidence of polycrine actions of prostaglandin E2. Endocrinology. 145(11):528-533.

5. ASHOWORTH, C.J.; BAZER, F.W. 1989. Changes in ovinus conceptus and endometrial function following asynchronous embryon transfer or administration of progesterone. Biol. of Reprod. 40:425-433.

6. ASSAL, M.A.; KINSKY, R.; MARTAL, J.; CHAOUAT, G. 1995. In vivo immunosuppressive effects of recombinant ovine interferon-tau (Trophoblastin): r.o.TP (r.o.IFN-T) inhibits local GVH reaction in mice (PLN assay), prevents fetal resorptions, and favors embryo survival and implantation in the $\mathrm{CBA} / \mathrm{J} \times \mathrm{DBA} / 2$ mice combination. Am. J. Reproduct. Immun. 33(3):267-275.

7. ASSELIN, E.; BAZER, F.W.; FORTIER, M.A. 1997. Recombinant ovine and bovine interferons tau regulate prostaglandin production and oxytocin response in cultured bovine endometrial cells. Biol. Reprod. 2:402-408.

8. ASSELIN, E.; GOFF, A.K.; BERGERON, H.; FORTIER, M.A. 1996. Influence of sex steroids on the production of prostaglandins F2 alpha and E2 and response to oxytocin in cultured epithelial and stromal cells of the bovine endometrium. Biol. Reprod. 2:371-379.

9. BILLIAU, A.; MATTHYS, P. 2009. Interferon-gamma: a historical perspective. Cytokine Growth Factor Rev. 20(2):97-113.
10. BINELLI, M.; SUBRAMANIAM, P.; DÍAZ, T.; JOHNSON, G.A.; HANSEN, T.R.; BADINGA, L.; THATCHER, W. 2001. Bovine interferon-tau stimulates the Janus kinase-signal transducer and activator of transcription pathway in bovine endometrial epithelial cells. Biol. of Reprod. 64(2):654-665.

11. CHARD, T. 1989. Fetal and maternal oxytocin in human parturition. J. Perinatol. 2:145-152.

12. CHEN, Y.; GREEN, J.A.; ANTONIOU, E.; EALY, A.D.; MATHIALAGAN, N.; WALKER, A.M.; AVALLE, M.P.; ROSENFELD, C.S.; HEARNE, L.B.; ROBERTS, R.M. 2006. Effect of interferon-tau administration on endometrium of nonpregnant ewes: a comparison with pregnant ewes. Endocrinology. 147(5):2127-2137.

13. CHENG, Z.; ABAYASEKARA, D.R.; WATHES, D.C. 2005. The effect of supplementation with $n-6$ polyunsaturated fatty acids on 1-, 2- and 3-series prostaglandin $\mathrm{F}$ production by ovine uterine epithelial cells. Biochim. Biophys. Acta. 2:128-135.

14. CHENG, Z.; ELMES, M.; KIRKUP, S.E.; ABAYASEKARA, D.R., WATHES, D.C. 2004. Alteration of prostaglandin production and agonist responsiveness by $n-6$ polyunsaturated fatty acids in endometrial cells from late-gestation ewes. J. Endocrinol. 2:249-256.

15. CHRISTINE, G.E.; ROSS, L.T.; KIM, C.W. 2009. The Genome Sequence of Taurine Cattle: A Window to Ruminant Biology and Evolution. Science. 324:522-528.

16. DEMARTINI, J.C.; CARLSON, J.O.; LEROUX, C.; SPENCER, T.; PALMARINI, M. 2003. Endogenous retroviruses related to jaagsiekta sheep retrovirus. Current Topics Microbiol. Immunol. 275:17-137.

17. DEMMERS, K.J.; DERECKA, K.; FLINT, A. 2001. Trophoblast interferon and pregnancy. Reproduction. 121(1):41-49.

18. EALY, A.D.; GREEN, J.A.; ALEXENKO, A.P.; KEISLER, D.H.; ROBERTS, R.M. 1998. Different ovine interferon-tau genes are not expressed identically and their protein products display different activities. Biol. of Reprod. 58(2):566-573. 
19. EZASHI, T.; EALY A.D.; OSTROWSKI, M.C.; ROBERTS, R.M. 1998. Control of interferon-tau gene expression by Ets-2. Proc. Nal. Acad. Sci. USA. 795(14):7882-7887.

20. FARINA, M.G.; BILLI, S.; LEGUIZAMÓN, G.; WEISSMANN, C.; GUADAGNOLI, T.; RIBEIRO, M.L.; FRANCHI, A.M. 2007. Secretory and cytosolic phospholipase A2 activities and expression are regulated by oxytocin and estradiol during labor. Reproduction. 2:355-364.

21. FORTIER, M.A.; GUILBAULT, L.A.; GRASSO, F. 1988. Specific properties of epithelial and stromal cells from the endometrium of cows. J. Reprod. Fertil. 1:239-248.

22. GEORGE, M.; LAMMING, G.E. 2001. Relationship between maternal endocrine environment, early embryon development and inhibition of the luteolytic machanism in cow. J. Reprod. Fertil. 121:175-180.

23. GÓMEZ, E.L.; BLANCO, M.; DOMÉNECH, A. 2007. Manual de Inmunología Veterinaria. Pearson Educación S.A. España. 728p.

24. GUZELOGLU, A.; BINELLI, M.; BADINGA, L.; HANSEN, T.R.; THATCHER, W. 2004a. Inhibition of phorbol ester-induced PGF2alpha secretion by IFN-tau is not through regulation of protein kinase C. Prostaglandins Other Lipid Mediat. 74(1-4):87-99.

25. GUZELOGLU, A.; SUBRAMANIAM, P.; MICHEL, F.; THATCHER, W. 2004b. Interferon-tau induces degradation of prostaglandin $\mathrm{H}$ synthase- 2 messenger RNA in bovine endometrial cells through a transcription-dependent mechanism. Biol. of Reprod. 71(1):170-176.

26. HASHIZUME, K.; SHIMADA, A.; NAKANO, H.; TAKAHASHI, T. 2006. Bovine trophoblast cell culture systems: a technique to culture bovine trophoblast cells without feeder cells. Methods Mol. Med. 121:179-188.

27. IMAKAWA, K.; HELMER, S.D.; NEPHEW, K.P.; MEKA, C.S.; CHRISTENSON, R.K. 1993. A novel role for GM-CSF: enhancement of pregnancy specific interferon production, ovine trophoblast protein-1. Endocrinology. 132(4):1869-1871.

28. IMAKAWA, K.; TAMURA, W.; MCGUIRE, J.; KHAN, S.L.; HARBISON, A.J.; STANGA, P.S.; HELMER, D.R.; CHRISTENSON, R.K. 1995. Effect of Inteleukin-3 on ovine trophoblast interferon during early conceptus. Develop. Endocrin. 3(7):511-517.

29. JEAN, M.F.; OMAR, C.; ERDOGAN, M. 2008. Culture systems for bovine embryos, Laboratory of Animal Functional Genomics. Dept. Animal and Dairy Science. Livestock Science. 121:141-149.

30. JO-ANN, G.; FLEMING, W.; YOUNGSOK, C.; GREG, A.J.; SPENCER, T.E.; BAZER, F.W. 2001. Cloning of ovine estrogen receptor-alpha promoter and functional regulation by ovine interferon tau. Endocrinology. 142:2879-2887.

31. KIM, S.; CHOI, Y.; SPENCER, T.; BAZER, F.W. 2003. Effects of the estrous cycle, pregnancy and interferon tau on expression of cyclooxygenase two (COX-2) in ovine endometrium. Reprod. Biol. Endocrinology. 20:1-58.

32. MARK, G.P.; LEE, S.D.; TINA, P.E.; KOJI, K.; CLIFTON, M.N.; JIM, W.E.; MONTY, K.S.; JONATHAN, G.A.; DUANE, K.H.; MICHAEL, R.R. 2008. Nutritional skewing of conceptus sex in sheep: effects of maternal diet enriched in rumen-protected polyunsaturated fatty acids (PUFA). Reprod. Biol. Endocrinol. 6:1-11.

33. OLIVERA, A.M.; TARAZONA, M.A.; RUIIZ, C.T.; GIRALDO, E.C. 2007. Modelo de luteólisis bovina. Rev. Col. Ciencias Pecuarias. 20:387-393.

34. OKUDA, K.; KASAHARA, Y.; MURAKAMI, S.; TAKAHASHI, H.; WOCLAWEK-POTOCKA, I.; SKARZYNSKI, D.J. 2004. Interferon-tau blocks the stimulatory effect of tumor necrosis factoralpha on prostaglandin F2alpha synthesis by bovine endometrial stromal cells. Biol. Reprod. 1:191-197.

35. PARENT, J.; VILLENEUVE, C.; ALEXENKO, A.P.; EALY, A.D.; FORTIER, M.A. 2003a. Influence of 
different isoforms of recombinant trophoblastic interferons on prostaglandin production in cultured bovine endometrial cells. Biol. of Reprod. 68(3):1035-1043.

36. PARENT, J.; VILLENEUVE, C.; FORTIER, M.A. 2003b. Evaluation of the contribution of cyclooxygenase 1 and cyclooxygenase 2 to the production of PGE2 and PGF2 alpha in epithelial cells from bovine endometrium. Reproduction. 4:539-547.

37. ROBERTS, R.M. 2007. Interferon-tau, a Type 1 interferon involved in maternal recognition of pregnancy. Cytokine Growth Factor Rev. 18(56):403-408.

38. ROBERTS, R.M.; YIZHEN, C.; TOSHIHIKO, E.; ANGELA, M.W. 2008. Interferons and the Maternalconceptus dialog in mammals. Review. Seminars in Cell y developmental Biology. 19:170-177.

39. ROBINSON, R.S.; PUSHPAKUMARA, P.G.; CHENG, Z.; PETERS, A.R.; ABAYASEKARA, D.R.; WATHES, D.C. 2002. Effects of dietary polyunsaturated fatty acids on ovarian and uterine function in lactating dairy cows. Reproduction. 1:119-131.

40. RODRÍGUEZ-SALLABERRY, C.; CALDARI-TORRES, C.; GREENE, E.S.; BADINGA, L. 2006. Conjugated linoleic acid reduces phorbol ester-induced prostaglandin F2alpha production by bovine endometrial cells. J Dairy Sci. 10:3826-3832.

41. SKARZYNSKI, D.J.; KOBAYASHI, S.; OKUDA, K. 2000a. Influence of nitric oxide and noradrenaline on prostaglandin $\mathrm{F}(2)$ (alpha)-induced oxytocin secretion and intracellular calcium mobilization in cultured bovine luteal cells. Biol. Reprod. 4:10001005.

42. SKARZYNSKI, D.J.; MIYAMOTO, Y.; OKUDA, K. 2000b. Production of prostaglandin $\mathrm{f}(2 \mathrm{alpha})$ by cultured bovine endometrial cells in response to tumor necrosis factor alpha: cell type specificity and intracellular mechanisms. Biol. Reprod. 5:1116-1120.

43. SKARZYNSKI, D.J.; PIOTROWSKA, K.; BAH, M.; KORZEKWA, A.; WOCLAWEK-POTOCKA, I.;
SAWAI, K.; OKUDA, K. 2008. Effects of exogenous tumour necrosis factor-alpha on the secretory function of the bovine reproductive tract depend on tumour necrosis factor-alpha concentrations. Reprod. Domest. Anim. 44(3):371-379.

44. SPENCER, T.E.; BAZER, F.W. 1996. Ovine interferon tau suppresses transcription of the estrogen receptor and oxytocin receptor genes in the ovine endometrium. Endocrinology. 3:1144-1147.

45. SPENCER, T.E.; BECKER, W.C.; GEORGE, P.; MIRANDO, M.A.; OGLE, T.F.; BAZER, F.W. 1995. Ovine interferon-tau regulates expression of endometrial receptors for estrogen and oxytocin but not progesterone. Biol. Reprod. 3:732-745.

46. SPENCER, T.E.; BURGHARDT, R.C.; JOHNSON, G.A.; BAZER, F.W. 2004. Conceptus signals for establishment and maintenance of pregnancy. Anim. Reprod. Sci. 82-83:537-550.

47. THATCHER, W.W.; STAPLES, C.R.; DANET, D.G.; OLDICK, B.; SCHMITT, E.P. 1994. Embryo health and mortality in sheep and cattle. J. Anim. Sci. 72:16-30.

48. TITHOF, P.K.; ROBERTS, M.P.; GUAN, W.; ELGAYYAR, M.; GODKIN, J.D. 2007. Distinct phospholipase A2 enzymes regulate prostaglandin E2 and F2 alfa production by bovine endometrial epithelial cells. Reprod. Biol. Endocrinology. 5:5-16.

49. WALKER, A.M.; KIMURA, K.; ROBERTS, R.M. 2009. Expression of bovine interferon-tau variants according to sex and age of conceptuses. Theriogenology. 72(1):44-53.

50. WEEMS, W.; WEEMS, Y.S.; RANDEL, R.D. 2006. Prostaglandins and reproduction in female farm animals. The Vet. J. 171:206-228.

51. WOCLAWEK, P.I.; DEPTULA, K.; BAH, M.M.; LEE, H.Y.; OKUDA, K.; SKARZYNSKI, D.J. 2004. Effects of nitric oxide and tumor necrosis factor-alpha on production of prostaglandin F2alpha and E2 in bovine endometrial cells. J. Reprod. Dev. 50(3):333-340. 
52. YAMAGUCHI, H.; NAGAOKA, K.; MATSUDA, F.; XU, $\quad$ Recibido: Agosto 14 de 2009 M.; CHRISTENSO, R.; IMAKAWA, K.; SAKI, S. Aceptado: Febrero 26 de 2010 2001. Regulation of interferon tau gene expression and the maternal recognition of pregnancy. J. Reprod. Dev. 47(2):69-82. 